\title{
Adenoma pleomórfico del espacio parafaríngeo: una entidad escondida
}

\section{Pleomorphic adenoma of the parapharyngeal space: a hidden entity}

\author{
Jai-sen Leung C. ${ }^{1}$, Rodrigo Aliaga D. ${ }^{1}$, Pablo Villanueva G. ${ }^{2}$, Claudio Callejas $C .{ }^{1}$
}

\section{Resumen}

Los tumores del espacio parafaríngeo son poco frecuentes. Dentro de ellos, las neoplasias benignas son las más frecuentes, destacando principalmente los adenomas pleomorfos. Estos tumores suelen permanecer asintomáticos hasta alcanzar el tamaño suficiente para producir alteraciones debido al efecto de masa. A pesar de su naturaleza benigna, es necesario realizar resección completa dada su alta tasa de recurrencia y potencial de transformación maligna. Presentamos el caso de un paciente de 52 años que en estudio de vértigo se detecta incidentalmente una masa tumoral del espacio parafaríngeo de $45 \mathrm{~mm}$ x $32 \mathrm{~mm}$ de diámetro, de etiología incierta. Se realizó una biopsia incisional endoscópica que evidenció un adenoma pleomórfico, que actualmente se encuentra en etapa de evaluación y planificación de escisión completa. En conclusión, dado que los hallazgos clínicos y radiológicos no siempre son suficientes para determinar la naturaleza de las lesiones del espacio parafaríngeo, es importante contar con un diagnóstico histológico. Los avances en las técnicas de cirugía endoscópica sinusal han permitido acceder a esta zona con una mínima morbilidad y estadía hospitalaria. Debido a la posibilidad de transformación maligna los adenomas pleomórficos requieren un manejo activo.

Palabras clave: espacio parafaríngeo, neoplasia, adenoma pleomórfico, estudio diagnóstico, tratamiento.

\begin{abstract}
Tumors of the parapharyngeal space are rare. Benign neoplasms are the most frequent and, among them, pleomorphic adenomas stand out. These tumors are usually asymptomatic until they reach a size large enough to produce symptoms due to mass effect. Despite its benign nature, it is necessary to perform a complete resection given its high recurrence rate and its potential for malignant transformation. We present the case of a 52-year-old man in whom a study of vertigo incidentally detected a mass in the parapharyngeal space measuring $45 \mathrm{~mm} \times 32 \mathrm{~mm}$ in diameter, of unknown etiology. An endoscopic incisional biopsy was performed, showing a pleomorphic adenoma, which is currently under assessment to plan complete removal. In conclusion, as clinical and radiological findings are not always sufficient to determine the nature of parapharyngeal space lesions, it is important to obtain a histological diagnosis. Advances in endoscopic sinus surgery techniques have allowed access to this area with minimal morbidity and hospital stay. Due to the possibility of malignant transformation pleomorphic adenomas require active management.
\end{abstract}

Keywords: parapharyngeal space, neoplasm, pleomorphic adenoma, diagnostic study, treatment.

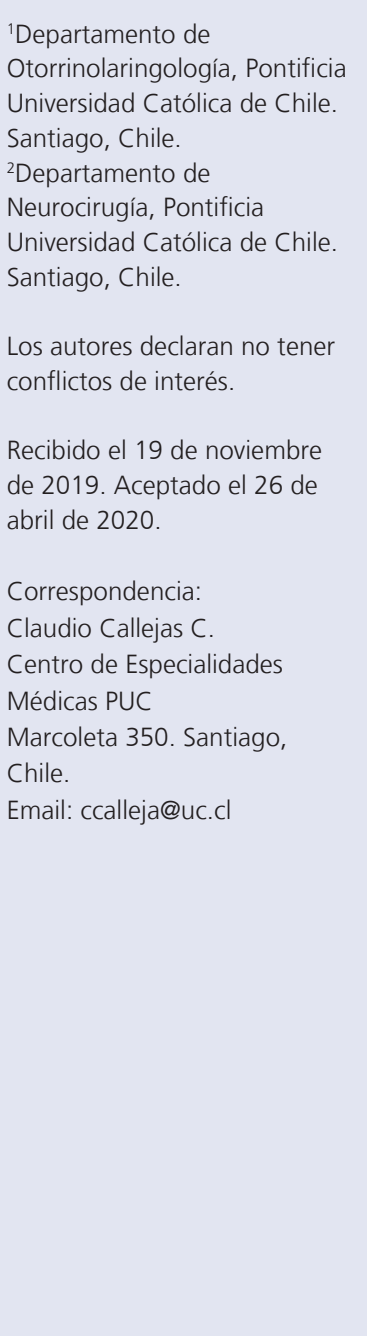

\section{Introducción}

El espacio parafaríngeo (EPF) corresponde a un espacio cervical suprahioideo que se extiende desde la base del cráneo hasta la hipofaringe. Presenta una forma de cono invertido, cuyo límite superior corresponde al hueso temporal. Su ápex o borde inferior es la unión del vientre posterior del músculo digástrico con el hueso hioides, mientras que 
el borde medial está delimitado por la fascia bucofaríngea y el borde lateral por la capa superficial de la fascia cervical profunda sobre el espacio masticatorio y el lóbulo profundo de la parótida. El borde anterior está delimitado por el rafe pterigomandibular y su borde posterior por la fascia prevertebral. La fascia tensor-vascular-estiloidea (TVS) divide a su vez al EPF en dos compartimientos: preestiloideo y retroestiloideo o espacio carotideo. El espacio preestiloideo alberga tejido adiposo y conectivo, además de ramas de la arteria y vena maxilar interna, ramas del trigémino y glándulas salivales menores. Mientras que el espacio retroestiloideo o carotídeo contiene la vaina carotídea con la arteria carótida interna y la vena yugular profunda, los nervios glosofaríngeos (IX), vago (X), accesorio (XI) e hipogloso (XII), la cadena simpática y algunos linfonodos ${ }^{1}$.

Los tumores del EPF son poco frecuentes, correspondiendo al 0,5\% de las neoplasias de cabeza y cuello. El 70\%-80\% corresponden a neoplasias benignas ${ }^{2}$, de ellas $50 \%$ son tumores de glándulas salivales, $41 \%$ neurogénicos y $9 \%$ presentan un origen distinto (tejido linfoide, muscular, metástasis, entre otros $)^{3}$. La mayoría de los pacientes con tumores en EPF consultan por la aparición de una masa en la zona cervical o la orofaringe, habiendo un porcentaje importante de pacientes en el que su diagnóstico corresponde a un hallazgo incidental en estudios imagenológicos ${ }^{4}$. Dentro de los tumores de EPF los adenomas pleomórficos (AP) son los más frecuentes, constituyendo el $40 \%$. La mayoría de ellos presenta un curso asintomático hasta alcanzar el tamaño suficiente para generar síntomas por efecto de masa. Sin embargo, a pesar de su naturaleza benigna, una parte de ellos puede presentar transformación maligna y recurrencia posterior al tratamiento ${ }^{5}$.

\section{Caso Clínico}

Paciente de 52 años, sexo masculino que consulta por síndrome vertiginoso. Dentro de evaluación diagnóstica se realiza resonancia magnética (RM) de encéfalo con angiorresonancia, que evidencia un tumor de bordes bien delimitados en espacio parafaríngeo izquierdo que mide $45 \mathrm{~mm}$ x $32 \mathrm{~mm}$ de diámetro en ejes axiales, señal hiperintensa en secuencia T2/ FLAIR con refuerzo sólido y múltiples áreas de degeneración quística en su interior, informado como posible schwanomma. Dicha lesión comprime la vena yugular, abomba la pared de la nasofaringe y altera la función de la trompa de Eustaquio, produciendo retención de secreciones otomastoideas ipsilaterales (Figura 1).

Dada la localización de la lesión se decide intentar biopsia endoscópica con apoyo de neuronavegación y monitorización de NC VII, IX, X y XI. Con el fin de obtener la mayor



Figura 1. RM de encéfalo con angiorresonancia. a: Corte axial RM secuencia T1. b: Corte sagital RM en secuencia STIR potenciada en T2. c: Corte axial RM en secuencia T2. Se observa lesión de 4,5 cm x 3,2 cm en espacio parafaríngeo izquierdo (flecha a) con áreas de degeneración quística en su interior. Espacio carotídeo y carótida izquierda comprimidos por masa tumoral (flecha b). Carótida derecha (flecha c). 
cantidad de reparos anatómicos (piso seno esfenoidal, órbita y base de cráneo) se realizó una fronto-esfeno-etmoidectomía y maxilectomía medial ipsilateral. Posteriormente, se resecó la pared posterior del seno maxilar y se abrió el periostio de la fosa pterigopalatina e infratemporal. Se identificó la apófisis pterigoides y tras desinsertar el músculo pterigoídeo lateral se siguió la lámina lateral de apófisis pterigoides hasta llegar a la lesión. Se identificó tumor blanquecino de consistencia dura y aspecto cartilaginoso (Figura 2).

Se envió muestra a biopsia intraoperatoria informada como tumor bifásico con componente escamoide. Dada la consistencia de la lesión y el informe intraoperatorio no concluyente de un diagnóstico definitivo, se decidió no intentar resección de la lesión hasta contar con biopsia definitiva. Se cubrió sitio quirúrgico con surgicel y no se dejó taponamiento

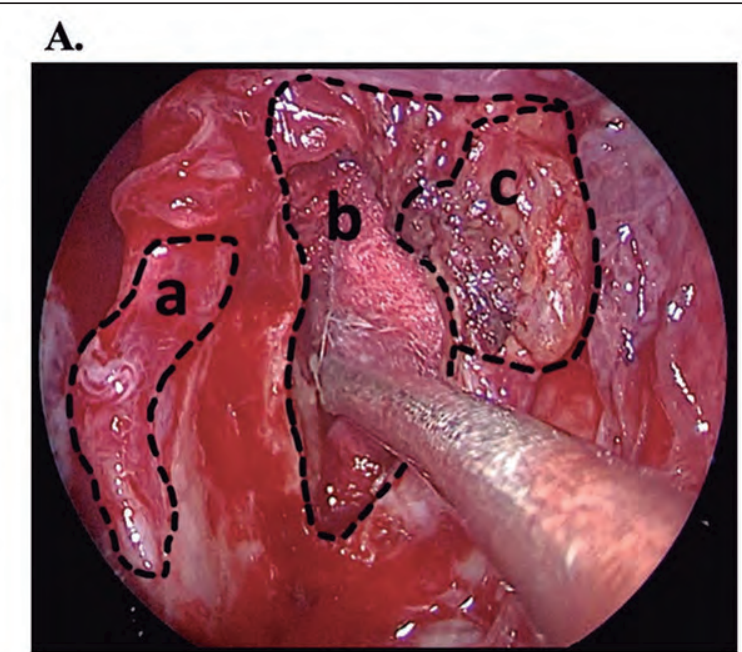

C.

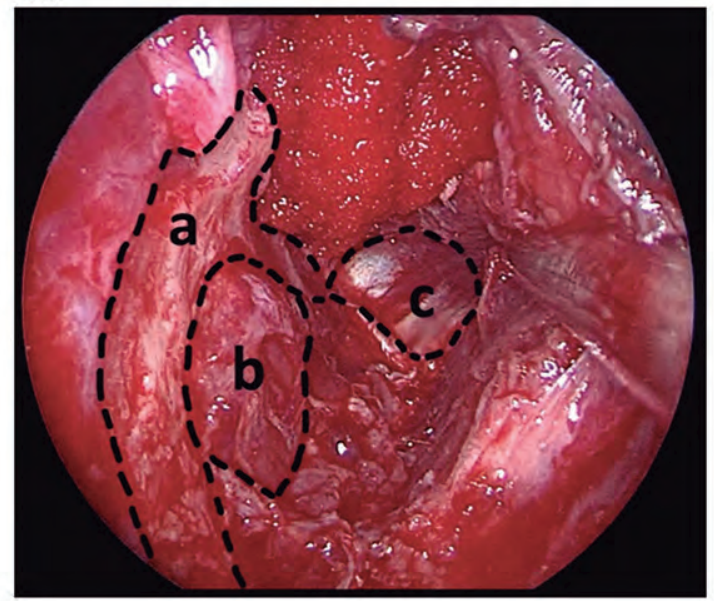

B.

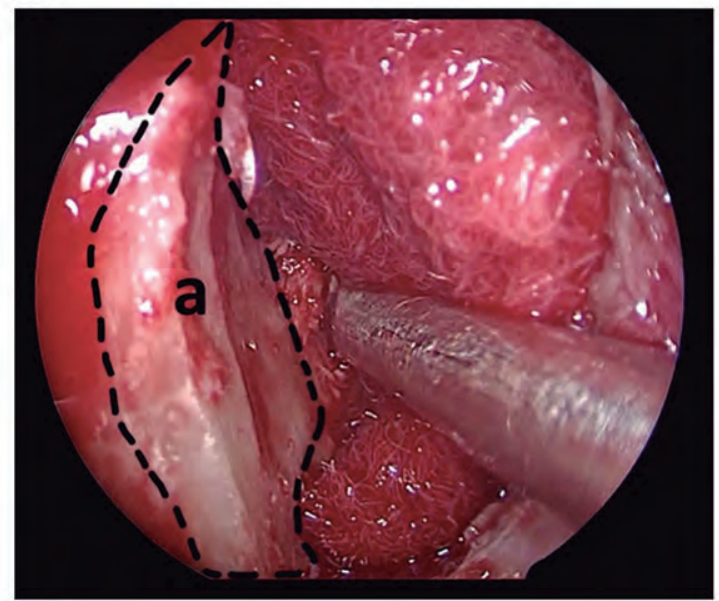

D.

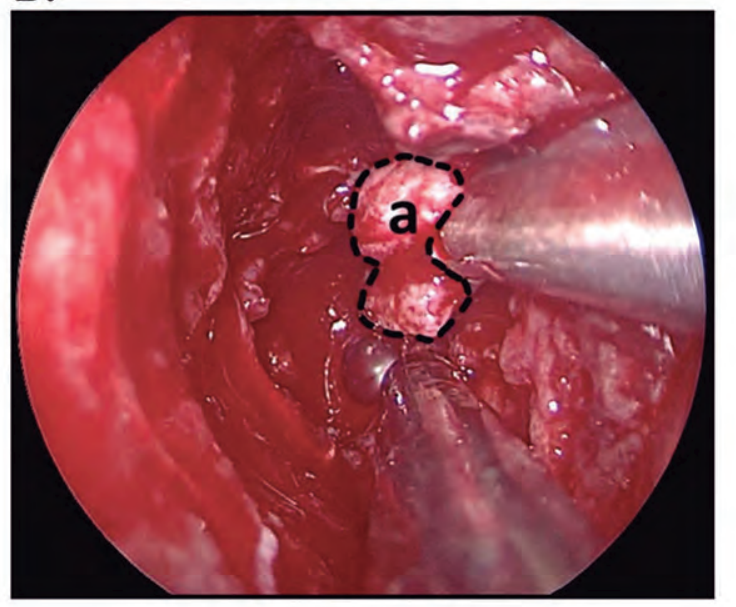

Figura 2. Imágenes intraoperatorias: abordaje, identificación de estructuras y obtención de muestras para biopsia. A. Visión endoscópica intraoperatoria: (a) Paquete vasculonervioso palatino. (b) Abertura de la pared posterior del seno maxilar. (c) Tejido adiposo de la fosa infratemporal. B. Visión endoscópica intraoperatoria con magnificación en apertura de pared posterior del seno maxilar: (a) Lámina pterigoidea lateral expuesta. C. Visión endoscópica intraoperatoria: (a) Lámina pterigoidea lateral expuesta y parcialmente resecada. (b) Músculo pterigoideo medial expuesto tras resección parcial de lámina lateral de apófisis pterigoides. (c) Masa tumoral en espacio parafaríngeo. D. Visión endoscópica intraoperatoria: (a) Muestra enviada a biopsia diferida. 
nasal. En el postoperatorio el paciente evolucionó favorablemente, por lo que fue dado de alta al día siguiente de la cirugía evolucionando satisfactoriamente en los controles sucesivos. El informe de la biopsia diferida evidenció un tumor bifásico, con doble grupo de población celular, sin signos de necrosis ni hemorragia. Se realizó estudio inmunohistoquímico que mostró el siguiente patrón de inmunorreactividad: P63, positivo en células mioepiteliales $(+)$; actina, positivo en células miopepiteliales $(+)$; S100, positivo $(+)$; citoqueratinas totales, positivo $(+)$; citoqueratina 7 , positivo $(+)$; índice de proliferación celular Ki-67, 5\%. Estos hallazgos fueron compatibles con un adenoma pleomórfico. Considerando los hallazgos histológicos y radiológicos, se concluyó que la lesión correspondía a un adenoma pleomórfico del lóbulo profundo de la parótida. Tras discusión en reunión multidisciplinaria se decidió realizar abordaje abierto con osteotomía mandibular de acceso para lograr resección completa de la lesión.

\section{Discusión}

Los tumores del EPF corresponden al 0,5\% de las neoplasias de cabeza y cuello. En nuestro país no existen reportes de incidencia de estas neoplasias. Sin embargo, distintas series internacionales concuerdan que la mayoría corresponden a tumores benignos $(74 \%-80 \%)^{6,7}$, siendo dentro de ellos los adenomas pleomorfos los más comunes (31\%-40\%), seguidos por los paragangliomas (35\%), schwannomas (16\%), ameloblastoma, neurofibromas y tumores de vasos sanguíneos. Por otro lado, el 20-30\% de las neoplasias del EPF son malignas, destacando las que provienen de glándulas salivales como las más frecuentes ${ }^{3,6,7}$. Los tumores del EPF suelen mantenerse asintomáticos hasta alcanzar un tamaño suficiente para causar síntomas; se ha descrito como límite $2,5 \mathrm{~cm}-3 \mathrm{~cm}$ para volverse sintomático ${ }^{8}$, pudiendo presentar: aumento de volumen cervical o intraoral (22\%$84 \%)$, otalgia (9\%-36\%), disfagia (12\%-15\%), disfunción tubaria (13\%), hipoacusia (5-11\%), disfonía (10\%), dolor facial $(6 \%)$, roncopatía (5\%), paresia del VII (3-4\%), IX (1\%), X (8\%) y XII par (5\%), odinofagia (2-8\%), tinnitus (2\%-5\%), parestesias linguales $(2 \%)$ y otitis media con efusión $(10 \%)^{3,7}$.
El estudio diagnóstico de estas neoplasias comprende un estudio imagenológico e histopatológico, el primero de ellos se realiza en base a tomografía computada (TC) o resonancia magnética, siendo esta última de mayor utilidad para definir el origen, extensión y vía de resección de la lesión? ${ }^{9}$ La presencia de desplazamiento del tejido adiposo hacia medial o anterior en las imágenes sugiere un origen neoplásico a partir del lóbulo profundo de la parótida, más aún, si la grasa se desplaza posteromedialmente probablemente exista extensión al EPF. Por otro lado, si el desplazamiento es hacia lateral, creando un plano graso entre el lóbulo profundo de la parótida y el tumor, es sugerente de un origen a partir de las glándulas salivales menores. El desplazamiento de la vena yugular y de la arteria carótida hacia posterior orienta a un origen desde el espacio preestiloideo, mientras que si la vena yugular es desplazada hacia posterior y/o lateral, asociado a un desplazamiento carotídeo anterior y/o medial, orientará a un origen en el espacio retroestiloídeo ${ }^{9-12}$.

En relación a los adenomas pleomórficos, corresponden a las neoplasias benignas más frecuentes de las glándulas salivales, siendo el 45\%-75\% de todos los tumores de glándulas salivales ${ }^{5}$. Su incidencia es de 2-3/100.000 habitantes, siendo más frecuentes en mujeres (2:1), pudiendo aparecer a cualquier edad ${ }^{13}$. Característicamente, el 84\% son dependientes de la glándula parótida, $8 \%$ de la submandibular y un $6,5 \%$ de las salivales menores. Histopatológicamente presentan células mioepiteliales y epiteliales, asociado a un aumento de marcadores de componentes estromales. Dentro de los hallazgos imagenológicos, a la TC se describen como una masa globular, lobulada, homogénea de densidad similar a los tejidos blandos con pocos focos de calcificación y en el caso de tumores de gran tamaño pueden observarse focos de necrosis al estudio contrastado. En el caso de la RM, aparecen como una masa bien delimitada, homogénea, en secuencia $\mathrm{T} 1$ tienen baja intensidad con realce uniforme al usar gadolinio, mientras que en T2 se ven hiperintensos con borde disminuido que representan la cápsula fibrosa circundante. $\mathrm{La}$ presencia de hipointensidad en T2 o bordes mal definidos al estudio contrastado debe hacer sospechar malignidad 5 . El tratamiento 
definitivo de estas lesiones es la resección total del tumor. De esta forma, si el tumor se origina en la glándula parótida se debe realizar una parotidectomía superficial o total, preservando el nervio facial. Por otro lado, si el tumor proviene de las glándulas submandibulares, debe realizarse la escisión de esta, preservando tanto las ramas del trigémino, hipogloso y el nervio lingual. Finalmente, si su origen es a partir de glándulas salivales menores se recomienda lograr un margen negativo de al menos $5 \mathrm{~mm}$ para evitar la recurrencia ${ }^{5}$. En cuanto al pronóstico, suele ser favorable en caso de realizarse la resección completa. Sin embargo, a pesar de lograr márgenes negativos un 7\%-15\% presenta recurrencia, volviéndose más resistentes al tratamiento. Adicionalmente, un porcentaje de estas lesiones sufre transformación maligna dependiente del tiempo entre el diagnóstico y la resección quirúrgica, reportándose tasas de 1,5\% en los primeros años y hasta 9,5\% después de 15 años 5 .

\section{Conclusión}

Los tumores del EPF son neoplasias poco frecuentes. Los tumores benignos suelen ser más frecuentes que los malignos y dentro de los primeros, los adenomas pleomórficos son los más comunes. El enfrentamiento de estas neoplasias se basa en la historia y examen clínico exhaustivo, asociado a un estudio imagenológico e histopatológico. En el caso de los adenomas pleomórficos, el tratamiento definitivo es su resección completa, dado las tasas de recurrencia y malignidad asociadas. De esta forma el diagnóstico y tratamiento precoz, asociado a seguimiento por un equipo multidisciplinario son fundamentales para lograr una sobrevida libre de enfermedad.

\section{Bibliografía}

1. Mallat N, Karma S, Zaghouani H, et al. Anatomy and common pathology of the parapharyngeal space: The imaging challenges. ECR 2015. Disponible en: http:// dx.doi.org/10.1594/ecr2015/C-0965.

2. Cabezas L, Cardemil F, Cabezas L. Tumores del espacio parafaríngeo: Revisión del tema. Rev Otorrinolaringol Cir Cabeza Cuello 2015; 75:187194. Disponible en https://doi.org/10.4067/S071848162015000200016.

3. van Hees T, van Weert S, Witte B, René Leemans C. Tumors of the parapharyngeal space: the VU University Medical Center experience over a 20-year period. Eur Arch Otorhinolaryngol 2018; 275:967-972. doi:10.1007/s00405-018-4891-x.

4. Suárez-Fente V, Llorente-Pendás J, GómezMartínez J, García-González L, López-Álvarez F, Suárez-Nieto C. Tumores primarios del espacio parafaríngeo. Nuestra experiencia en 51 pacientes. Acta Otorrinolaringol Esp 2009; 60:19-24. Disponible en https://doi.org/10.1016/S0001-6519(09)70313-9.

5. Bokhari MR, Greene J. Pleomorphic Adenoma. StatPearls Treasure Island. Disponible en: https:// www.ncbi.nlm.nih.gov/books/NBK430829.

6. Grilli G, Suárez V, Muñoz M, Costales M, Llorente J. Tumores primarios del espacio parafaríngeo. Acta Otorrinolaringol Esp 2017; 68:138-144. Disponible en https://doi.org/10.1016/j.otorri.2016.06.003.

7. Hughes K, Olsen K, McCaffrey T. Parapharyngeal space neoplasms. Head Neck 1995;17:124-130. Doi: 10.1002/hed.2880170209.

8. Dimitrijevic MV, Jesic SD, Mikic AA, Arsovic NA, Tomanovic NR. Parapharyngeal space tumors: 61 case reviews. Int J Oral Maxillofac Surg 2010; 39:983-989. Disponible en https://doi.org/10.1016/j. ijom.2010.06.005.

9. Chen Z, Chen Y, Yu Q, et al. Excision of tumors in the parapharyngeal space using an endoscopically assisted transoral approach: a case series and literature review. J Int Med Res 2018; 47:1103-1113. Disponible en https://doi.org/10.1177/0300060518816190.

10. Iseri M, Ozturk M, Kara A, Ucar S, Aydin O, Keskin G. Endoscope-assisted transoral approach to parapharyngeal space tumors. Head Neck 2015;37:243-248. Disponible en https://doi. org/10.1002/hed.23592.

11. Stambuk H, Patel S. Imaging of the Parapharyngeal Space. Otolaryngol Clin North Am 2008; 41:77101. Disponible en https://doi.org/10.1016/j. otc.2007.10.012.

12. Som P, Sacher M, Stollman A, Biller H, Lawson $\mathrm{W}$. Common tumors of the parapharyngeal space: refined imaging diagnosis. Radiology 1988;169:8185. Disponible en https://doi.org/10.1148/ radiology.169.1.2843942.

13. Zhan KY, Khaja SF, Flack AB, Day TA. Benign Parotid Tumors. Otolaryngol Clin North Am 2016;49:327-342. Disponible en https://doi. org/10.1016/j.otc.2015.10.005. 